\title{
Genetic Variability of Antioxidative Mechanisms and Cardiotoxicity after Adjuvant Radiotherapy in HER2-Positive Breast Cancer Patients
}

\author{
Tanja Marinko $\mathbb{D},,^{1,2}$ Jakob Timotej Stojanov Konda $\left(\mathbb{D},{ }^{2}\right.$ Vita Dolžan $\left(\mathbb{D},{ }^{3}\right.$ \\ and Katja Goričar iD ${ }^{3}$ \\ ${ }^{1}$ Institute of Oncology Ljubljana, Ljubljana, Slovenia \\ ${ }^{2}$ University of Ljubljana, Faculty of Medicine, Ljubljana, Slovenia \\ ${ }^{3}$ University of Ljubljana, Faculty of Medicine, Institute of Biochemistry and Molecular Genetics, Pharmacogenetics Laboratory, \\ Ljubljana, Slovenia
}

Correspondence should be addressed to Katja Goričar; katja.goricar@mf.uni-lj.si

Received 8 October 2020; Revised 2 November 2020; Accepted 9 December 2020; Published 22 December 2020

Academic Editor: Anca Daniela Farcaş

Copyright (C) 2020 Tanja Marinko et al. This is an open access article distributed under the Creative Commons Attribution License, which permits unrestricted use, distribution, and reproduction in any medium, provided the original work is properly cited.

\begin{abstract}
Background. Breast cancer treatment is associated with the occurrence of various cardiac adverse events. One of the mechanisms associated with cardiotoxicity is oxidative stress, against which cells are protected by antioxidative enzymes. Genetic variability of antioxidative enzymes can affect enzyme activity or expression, which modifies the ability of cells to defend themselves against oxidative stress and could consequently contribute to the occurrence of treatment-related cardiotoxicity. Our aim was to evaluate the association of common polymorphisms in antioxidative genes with cardiotoxicity after adjuvant radiotherapy (RT) in HER2-positive breast cancer patients. Methods. Our retrospective study included 101 HER2-positive early breast cancer patients who received trastuzumab and adjuvant RT. We isolated DNA from buccal swabs and used competitive allele-specific PCR for genotyping of PON1 rs854560 and rs662, GSTP1 rs1138272 and rs1695, SOD2 rs4880, CAT rs1001179, and HIF1 rs1154965 polymorphisms. N-terminal pro B-type natriuretic peptide (NT-proBNP), left ventricular ejection fraction, and NYHA class were used as markers of cardiotoxicity. We used logistic regression to evaluate the association of genetic factors with markers of cardiotoxicity. Results. Carriers of at least one polymorphic PON1 rs854560 allele were less likely to have increased NT-proBNP $(\mathrm{OR}=0.34 ; 95 \% \mathrm{CI}=0.15-0.79 ; P=0.012)$, even after adjustment for age $(\mathrm{OR}=0.35 ; 95 \% \mathrm{CI}=0.15-0.83$; $P=0.017)$. Carriers of at least one polymorphic PON1 rs662 allele were more likely to have increased NT-proBNP $(\mathrm{OR}=4.44$; 95\% $\mathrm{CI}=1.85-10.66 ; P=0.001)$, even after adjustment for age $(\mathrm{OR}=5.41 ; 95 \% \mathrm{CI}=2.12-13.78 ; P<0.001)$. GSTP1 rs1695 was also associated with decreased NT-proBNP in the multivariable analysis $(P=0.026)$, while $C A T$ rs 1001179 was associated with NYHA class in the univariable $(P=0.012)$ and multivariable analysis $(P=0.023)$. Conclusion. In our study, polymorphisms PON1 rs662 and rs854560, CAT rs1001179, and GSTP1 rs1695 were significantly associated with the occurrence of cardiac adverse events after adjuvant RT and could serve as biomarkers contributing to treatment personalization.
\end{abstract}

\section{Introduction}

Adjuvant radiotherapy (RT) has significantly improved disease-specific survival for patients with early-stage breast cancer $[1,2]$. As a consequence, more cancer survivors may experience late complications of treatment [3]. Radiation dose received by the heart during adjuvant RT of breast or thoracic wall may result in a range of cardiotoxic effects including coronary artery disease, cardiomyopathy, pericardial disease, valvular dysfunction, and conduction abnormalities $[4,5]$. There is no minimum radiation dose to the heart that is entirely safe [4].

A combination of adjuvant RT and systemic oncological treatment may have an even worse impact on the cardiac- 
related outcome [6]. This combination is frequently used in HER2-positive breast cancer, a subtype of breast cancer with amplification or overexpression of the human epidermal growth factor receptor 2 (HER2) oncogene, which represents approximately $15 \%$ of all breast cancers $[7,8]$. In standard clinical practice, this subtype of breast cancer is treated with a least two types of cardiotoxic systemic treatment [7, 9]. Anthracyclines are prescribed as a part of neoadjuvant or adjuvant chemotherapy and are followed by anti-HER2 treatment with trastuzumab, a monoclonal antibody [9]. Both types of treatment increase the survival of HER2positive breast cancer patients but are cardiotoxic [10-13]. Anthracycline-related cardiotoxicity results, at least to some degree, in myocyte destruction and clinical heart failure and is irreversible. Trastuzumab-related cardiotoxicity is most often manifested by an asymptomatic decrease in left ventricular ejection fraction (LVEF) and less often by clinical heart failure [13-15].

Different biomarkers and imaging techniques and their potential role in monitoring cardiotoxicity have already been evaluated [6]. The use of blood-based biomarkers to detect radiation or systemic treatment-induced cardiotoxicity is very promising as it is minimally invasive, affordable, and repeatable [16]. Currently, the determination of LVEF and $\mathrm{N}$-terminal pro-brain natriuretic peptide (NT-proBNP) is mostly used for monitoring cardiotoxicity of cancer treatment $[14,17,18]$. LVEF is the golden standard for monitoring cardiac function in patients receiving cardiotoxic therapy. Echocardiography and radionuclide ventriculography are imaging techniques that are being most widely used in this setting for the assessment of LVEF [14].

Brain natriuretic peptide (BNP), a member of the family of natriuretic hormones, seems to be one of the most appropriate biomarkers for cardiotoxicity evaluation $[19,20]$. After being synthesized, its inactive form is then cleaved into active BNP and inactive NT-proBNP. NT-proBNP is a sensitive biomarker of both systolic and diastolic heart failure [21]. NT-proBNP was also an early and sensitive diagnostic and prognostic biomarker for the evaluation of cardiotoxicity of cancer chemotherapy and RT [20, 22, 23]. Patients with elevated NT-proBNP had a higher possibility of asymptomatic LVEF reduction or developing symptomatic heart failure later on. Because changes in NT-proBNP usually occur earlier than changes in LVEF, its elevated level exposes patients at higher risk.

One of the molecular mechanisms associated with cancer treatment response and occurrence of adverse events is oxidative stress. Oxidative stress occurs as a result of excess formation of reactive oxygen species (ROS) that can cause a variety of cellular damage, including DNA modifications, breaks, deletions and translocations, lipid peroxidation, amino acid modifications, and protein conformational changes [24]. Oxidative stress is also among the key stimuli leading to carcinogenesis [25]. RT can trigger oxidative stress in cancer cells, causing DNA damage and stress response activation in mitochondria and the endoplasmic reticulum. Increased ROS formation affects both cancer and surrounding healthy cells, leading to various adverse events, including cardiotoxicity [26, 27]. Oxi- dative stress was also proposed as one of the mechanisms involved in anthracycline and trastuzumab cardiotoxicity [28-30]. Anthracyclines increase ROS formation through various enzymatic and nonenzymatic reactions $[28,30]$. For example, the reduction of anthracyclines results in the formation of semiquinone free radicals and increased formation of ROS through different enzymes. Anthracyclines also interact with ferric iron, leading to altered redox-cycling and the formation of superoxide anion [30]. Trastuzumab is associated with increased ROS formation, decreased glutathione concentration, and decreased activity of antioxidative enzymes in cell lines [31]. Studies suggest trastuzumab mostly affects oxidative stress due to dysregulated HER2 signalling through mitogen-activated protein kinase, phosphoinositide 3-kinase, and neuregulin signalling pathways leading to increased ROS formation $[29,30]$.

Antioxidative enzymes are part of the cellular mechanisms maintaining appropriate levels of ROS and could therefore affect treatment-related cardiotoxicity. Among them are glutathione-S-transferases (GSTs), crucial for detoxification of endogenous and exogenous substrates by conjugation with reduced glutathione. The most important subtypes are GSTM1, GSTT1, and GSTP1 [32]. The enzyme superoxide dismutase (SOD) catalyzes the conversion of superoxide radical to hydrogen peroxide [33], after which the latter can be converted to water, catalyzed by the enzyme catalase [34]. Another antioxidative enzyme is paraoxonase 1 (PON1) that has organophosphates, lactonase, and esterase activity and is located in high-density lipoproteins (HDL) [35]. Oxidative stress was also proposed as a modulator of hypoxia-inducible factor 1 (HIF1) activity [36, 37], a transcription factor involved in response to hypoxia that regulates the expression of several genes involved in important cell processes and diseases $[38,39]$.

There are significant differences in the occurrence of postirradiation toxicity among breast cancer patients, and genetic factors may contribute to the observed interindividual variability [40-42]. Several genetic polymorphisms affect the activity or expression of antioxidative enzymes [32, 39, 43-45] and could consequently also affect the occurrence of treatment-related cardiotoxicity.

The aim of our study was therefore to evaluate whether common polymorphisms in antioxidative genes affect the cardiotoxicity after adjuvant RT in HER2-positive early breast cancer patients.

\section{Patients and Methods}

2.1. Patients. Our retrospective study included patients with human epidermal growth factor receptor-2- (HER2-) positive left- or right-sided early breast cancer (stage I-III), treated concurrently with trastuzumab and RT at the Institute of Oncology Ljubljana between June 2005 and December 2010. HER2 status of the tumour was determined according to our standard clinical practice [8]. All patients were treated according to the clinical guidelines with surgery, chemotherapy, endocrine therapy in case of hormone receptor-positive disease, trastuzumab, and RT. Trastuzumab treatment started before RT or on the first day of RT at the latest. After 
the adjuvant treatment with RT and trastuzumab, a followup clinical examination was performed. All patients also filled out questionnaires about smoking, concomitant diseases, and problems related to cardiovascular diseases. All other data were obtained from the patients' records.

The study was approved by the Republic of Slovenia National Medical Ethics Committee (approval number $39 / 05 / 15,0120-54 / 2015-2)$ and was internationally registered at ClinicalTrial.gov (identifier NCT01572883). The study was conducted in accordance with the Declaration of Helsinki. All participants signed an informed consent before participating in the study.

2.1.1. Systemic Treatment. Data regarding systemic therapy were obtained from the patient's individual medical record. Most patients were treated with one of the chemotherapy regimens that include anthracyclines and taxanes and were used in standard clinical practice at the time of the treatment. Mostly used treatment schemes were as follows: Option 1: 4 cycles of epirubicin plus cyclophosphamide (EC) or doxorubicin plus cyclophosphamide (AC) every 3 weeks, followed by 12 cycles of paclitaxel weekly; Option 2: 4 cycles of EC or AC every 3 weeks, followed by 3 cycles of docetaxel every 3 weeks; or Option 3: 3 to 4 cycles of 5-fluorouracil, epirubicin, and cyclophosphamide (FEC) or doxorubicin in combination with 5-fluorouracil and cyclophosphamide (FAC) every 3 weeks, followed by 3-4 cycles of docetaxel every 3 weeks. The criteria for adjuvant treatment with trastuzumab regarding tumour, nodal stage, and cardiac function were the same as in pivotal adjuvant trials: tumours larger than $2 \mathrm{~cm}$ if node-negative disease, any tumour size if node-positive disease, WHO performance status zero or one, no serious concomitant cardiac disease, and treatment with adjuvant chemotherapy [46]. Treatment with trastuzumab started 3 weeks after the last cycle of anthracyclines and was prescribed for 1 year.

2.1.2. Locoregional Treatment. According to clinical guidelines patients were operated with either breast conservation surgery or mastectomy and either sentinel node biopsy or axillary dissection. After the operation and chemotherapy, they were irradiated using two-dimensional (2D RT) or three-dimensional conformal RT (3D CRT). Some of the patients received electron-beam chest wall irradiation. Whole breast RT was required in all patients who underwent breast cancer surgery. In addition to the irradiation of the breast/chest wall, all patients with 4 or more positive axillary lymph nodes also received regional RT.

Patients were irradiated with a total dose $(\mathrm{TD})=25 \times$ $2 \mathrm{~Gy}, 5$ fractions per week. A minority received RT with $\mathrm{TD}=17$ or $18 \times 2.5 \mathrm{~Gy}, 5$ fractions per week. RT was performed 3 or more weeks after chemotherapy had been completed and concurrently with trastuzumab treatment as well as hormonal therapy in case of hormone receptor-positive breast cancer.

2.2. Assessment of Cardiotoxicity. Patients were classified according to New York Heart Association (NYHA) classification to assess signs of heart failure [47].
2.2.1. Echocardiography and Radionuclide Ventriculography. Echocardiography with LVEF measurement was performed before adjuvant RT and after the completed treatment with RT and trastuzumab. Baseline LVEF was determined either by using echocardiography or radionuclide ventriculography as previously described [8]. Normal range for LVEF was 50\% or more. The difference between both LVEF measurements was analysed. Absolute change in LVEF was calculated as the difference between LVEF after completed adjuvant RT and trastuzumab treatment, and LVEF before RT. Important LVEF reduction was classified as a decrease of LVEF for $\geq 10 \%$ or a final value of $\mathrm{LVEF}<50 \%$ [17].

2.2.2. NT-proBNP. NT-proBNP was determined with the Cobas e 411 analyser (Roche) according to our standard clinical practice at the follow-up clinical examination after the adjuvant treatment with RT and trastuzumab [8]. According to the instructions of the manufacturer, the values of NTproBNP below $125 \mathrm{ng} / \mathrm{l}$ exclude heart dysfunction [48].

2.3. DNA Extraction and Genotyping. Genomic DNA was extracted from buccal swabs (INFINITI Buccal Sample Collection Kit, AutoGenomics Inc., Vista, CA, USA) using QIAamp DNA Mini Kit (QIAGEN, Hilden, Germany) according to the manufacturer's instructions. Common putatively functional single nucleotide polymorphisms (SNPs) in antioxidative genes GSTP1, CAT, SOD2, PON1, and HIF1 were selected based on literature search. Genotyping was performed using fluorescent-based competitive allele-specific polymerase chain reaction (KASP, LGC Genomics, UK) following the manufacturer's instructions.

2.4. Statistical Analysis. Continuous and categorical variables were described using median with interquartile range $(25 \%-$ $75 \%)$ and frequencies, respectively. A dominant genetic model was used in the analyses. Deviation from HardyWeinberg equilibrium (HWE) was evaluated using chisquare test, and SNPs not in HWE were excluded from further analyses. To evaluate the association of selected SNPs with markers of cardiotoxicity, univariable and multivariable logistic regression were used to calculate odds ratios (ORs) and $95 \%$ confidence intervals (CIs). Clinical parameters used for adjustment were selected using stepwise forwardconditional logistic regression. Fisher's exact test was used to compare genotype frequencies if there were no patients in one of the categories. The nonparametric Mann-Whitney test was used to compare the distribution of continuous variables. The statistical analyses were carried out by using IBM SPSS Statistics version 21.0 (IBM Corporation, Armonk, NY, USA). To determine the combined effect of more SNPs within one gene, we reconstructed haplotypes using Thesias with the most common haplotype serving as a reference [49]. All statistical tests were two-sided, and the level of significance was set at 0.05 .

\section{Results}

We included 101 HER2-positive early breast cancer patients treated with adjuvant RT and trastuzumab. Regarding systemic treatment, $99(98.0 \%)$ patients were also treated with 
anthracyclines. Additionally, 58 (57.4\%) received taxanes and $57(56.4 \%)$ received hormonal therapy. Median time between the first and the last anthracycline application was 67 (51-105) days. Median time between the first and the last taxane application was 43 (42-75) days. Most patients (84, $83.2 \%$ ) were irradiated with a total dose of $25 \times 2 \mathrm{~Gy}, 5$ fractions per week. All treatment was administered according to clinical guidelines. A total of 48 (47.5\%) patients were operated and received RT on the left side. Detailed patients' characteristics and treatment parameters are presented in Table 1.

Median follow-up after diagnosis was 4.5 (3.2-5.9) years, and median follow-up after the onset of RT was 4.0 (2.6-5.4) years. Cardiotoxicity was evaluated in all patients after the treatment (Table 2). Median NT-proBNP level was 90 (56157) ng/l. In total, 36 (35.6\%) patients had increased NTproBNP values with above $125 \mathrm{ng} / \mathrm{l}$. Most patients did not exhibit signs of heart failure according to NYHA classification. After treatment, 17 (16.8\%) patients had mild symptoms (NYHA class 2). LVEF measurements before and after RT were similar with the median change of 3 (-3 to 9$) \%$. Important LVEF reduction was observed in $9(8.9 \%)$ patients (Table 2).

All patients were genotyped for PON1 rs854560 (p.Leu55Met), PON1 rs662 (p.Gln192Arg), GSTP1 rs1138272 (p.Ala114Val), GSTP1 rs1695 (p.Ile105Val), SOD2 rs4880 (p.Ala16Val), CAT rs1001179 (c.-330C>T), and HIF1A rs1154965 (p.Pro582Ser). Genotype and minor allele frequencies are presented in Supplementary Table 1. As SOD2 rs4880 genotype distribution was not in agreement with HWE $(P=0.004)$, this SNP was excluded from further analyses. Genotype distributions of all other SNPs were in agreement with HWE.

3.1. NT-proBNP. Carriers of at least one polymorphic PON1 rs854560 allele had significantly lower median NT-proBNP level $(P=0.048$, Table 3, Figure $1(\mathrm{a}))$, while carriers of at least one polymorphic PON1 rs662 allele had significantly higher median NT-proBNP level $(P=0.007$, Table 3 , Figure $1(b))$.

Among clinical parameters, only higher age was significantly associated with increased NT-proBNP in our study group $(\mathrm{OR}=0.61,95 \% \mathrm{CI}=0.27-1.38, P=0.231)$. Surgical treatment and RT side was not associated with increased NT-proBNP (right vs. left: $\mathrm{OR}=1.05,95 \% \mathrm{CI}=1.01-1.09, P$ $=0.023$ ). None of the other treatment parameters, including chemotherapy parameters, or comorbidities were significantly associated with NT-proBNP (all $P>0.05$ ).

Carriers of at least one polymorphic PON1 rs854560 allele were significantly less likely to have increased NTproBNP $(\mathrm{OR}=0.34,95 \% \mathrm{CI}=0.15-0.79, P=0.012)$, even after adjustment for age $(\mathrm{OR}=0.35,95 \% \mathrm{CI}=0.15-0.83, P$ $=0.017$ ) (Table 3 ). Carriers of at least one polymorphic PON1 rs662 allele were significantly more likely to have increased NT-proBNP $(\mathrm{OR}=4.44,95 \% \mathrm{CI}=1.85-10.66, P$ $=0.001)$. This association remained significant even after adjustment for age $(\mathrm{OR}=5.41,95 \% \mathrm{CI}=2.12-13.78, P<$ $0.001)$. Additionally, carriers of at least one polymorphic GSTP1 rs1695 were less likely to have increased NT-proBNP, but this difference was significant only after adjustment for age $(\mathrm{OR}=0.36,95 \% \mathrm{CI}=0.15-0.88, P=0.026)$.
To evaluate the combined effect of both PON1 SNPs on NT-proBNP, haplotype analysis was performed. Three haplotypes were observed in our study: PON1 TA, AA in AG (SNP order from $5^{\prime}$-end to $3^{\prime}$-end: rs854560, rs662) and their estimated frequencies were $0.366,0.366$, and 0.267 , respectively. Compared to reference PON1 TA haplotype, carriers of PON1 AG haplotype were significantly more likely to have increased NT-proBNP (OR $=5.48,95 \%$ $\mathrm{CI}=2.10-14.29, P<0.001)$. On the other hand, PON1 AA haplotype was not associated with NT-proBNP $(\mathrm{OR}=1.33$, 95\% CI $=0.66-2.69, P=0.418)$.

3.2. LVEF. Operation and RT side was not associated with LVEF reduction (right vs. left: $\mathrm{OR}=1.15,95 \% \mathrm{CI}=0.29$ $4.54, P=0.846)$. Other clinical characteristics, including chemotherapy parameters, were also not associated with LVEF reduction in our study (all $P>0.05$ ). None of the investigated SNPs was associated with LVEF (Table 4).

3.3. NYHA. Among clinical parameters, higher NYHA class was associated with higher body mass index (BMI) $(\mathrm{OR}=1.20,95 \% \mathrm{CI}=1.05-1.38, P=0.006)$ and presence of hyperlipidemia $(\mathrm{OR}=4.60,95 \% \mathrm{CI}=1.39-15.19, P=0.012)$. Operation and RT side was not associated with NYHA class (right vs. left: $\mathrm{OR}=0.77,95 \% \mathrm{CI}=0.27-2.19, P=0.624$ ). Other clinical characteristics, including chemotherapy parameters, were also not associated with NYHA class in our study (all $P>0.05$ ).

Carriers of at least one polymorphic CAT rs1001179 allele were significantly more likely to be NYHA class 2 (OR $=4.09,95 \% \mathrm{CI}=1.37-12.25, \quad P=0.012)$, even after adjustment for hyperlipidemia and BMI $(\mathrm{OR}=4.14,95 \%$ $\mathrm{CI}=1.22-14.09, P=0.023)$. Other SNPs were not associated with NYHA class in univariable or multivariable analysis (Table 4).

Among patients with left-sided breast cancer only, 9 (18.8\%) patients were NYHA class 2. Carriers of at least one polymorphic $C A T$ rs 1001179 allele were still significantly more likely to be NYHA class $2(\mathrm{OR}=5.09,95 \% \mathrm{CI}=1.08$ 24.02, $P=0.040$ ) in univariable analysis, while only a trend was observed after adjustment for hyperlipidemia and BMI (OR $=5.94,95 \% \mathrm{CI}=0.84-42.22, P=0.075)$. Other SNPs were also not associated with NYHA class in univariable or multivariable analysis in left-sided breast cancer (all $P>$ $0.05)$.

\section{Discussion}

In the present study, we evaluated the association of genetic variability in antioxidative genes with cardiotoxicity in HER2-positive early breast cancer patients treated with adjuvant RT and trastuzumab. We showed that PON1 rs854560 and rs662 as well as GSTP1 rs1695 polymorphisms were associated with NT-proBNP levels, while CAT rs1001179 was associated with NYHA class.

Cardiotoxicity of breast cancer treatment has been widely investigated in recent years as improvements in cancer treatment that led to improved long-term survival also increased treatment-related cardiotoxicity [18]. Both systemic therapy 
TABLE 1: Characteristics of breast cancer patients included in the study $(N=101)$ and treatment parameters.

\begin{tabular}{|c|c|c|}
\hline Characteristic & Category/Unit & $N(\%)$ \\
\hline Age & Years & $\begin{array}{c}50.9 \\
(42.1-59.1)^{*}\end{array}$ \\
\hline \multirow{2}{*}{ Type of surgery } & Mastectomy & $48(47.5)$ \\
\hline & Conservative surgery & $53(52.5)$ \\
\hline \multirow{2}{*}{ Side of surgery } & Left & $48(47.5)$ \\
\hline & Right & $53(52.5)$ \\
\hline \multirow{3}{*}{ Tumour type } & Invasive lobular carcinoma & $2(2.0)$ \\
\hline & Invasive ductal carcinoma & $96(95.0)$ \\
\hline & Other & $3(3.0)$ \\
\hline \multirow{3}{*}{ Cancer grade } & 1 & $1(1.0)$ \\
\hline & 2 & $31(30.7)$ \\
\hline & 3 & $69(68.3)$ \\
\hline \multirow{3}{*}{$\begin{array}{l}\text { Chemotherapy } \\
\text { scheme }\end{array}$} & $\mathrm{AC} / \mathrm{EC} / \mathrm{FAC} / \mathrm{FEC}$ with taxanes & $54(53.5)$ \\
\hline & $\begin{array}{c}\text { AC/EC/FAC/FEC without } \\
\text { taxanes }\end{array}$ & $43(42.6)$ \\
\hline & Other & $4(4.0)$ \\
\hline \multirow{4}{*}{ Anthracyclines } & Yes & $99(98.0)$ \\
\hline & Doxorubicin & $6(6.0)$ \\
\hline & Epirubicin & $93(92.1)$ \\
\hline & No & $2(2.0)$ \\
\hline \multirow{2}{*}{$\begin{array}{l}\text { Anthracyclines } \\
\text { cumulative dose }\end{array}$} & Doxorubicin, mg/m² BSA & $\begin{array}{c}342 \\
(318-413)^{*}\end{array}$ \\
\hline & Epirubicin, $\mathrm{mg} / \mathrm{m}^{2} \mathrm{BSA}$ & $\begin{array}{c}353 \\
(294-522)^{*}\end{array}$ \\
\hline \multirow{4}{*}{ Taxanes } & Yes & $58(57.4)$ \\
\hline & Paclitaxel & $17(16.7)$ \\
\hline & Docetaxel & $41(40.6)$ \\
\hline & No & $43(42.6)$ \\
\hline \multirow{2}{*}{$\begin{array}{l}\text { Taxanes } \\
\text { cumulative dose }\end{array}$} & Paclitaxel, mg/m² BSA & $\begin{array}{c}886 \\
(739-938)^{*}\end{array}$ \\
\hline & Docetaxel, mg/m² BSA & $\begin{array}{c}286 \\
(269-299) *\end{array}$ \\
\hline \multirow{2}{*}{ Hormonal therapy } & Yes & $57(56.4)$ \\
\hline & No & $44(43.6)$ \\
\hline \multirow{2}{*}{$\begin{array}{l}\text { Treatment scheme } \\
\text { of RT }\end{array}$} & $25 \times 2 \mathrm{~Gy}$ & $84(83.2)$ \\
\hline & 17 or $18 \times 2.5 \mathrm{~Gy}$ & $17(16.8)$ \\
\hline \multirow[b]{2}{*}{ Site of RT } & Breast/mammary region & $58(57.4)$ \\
\hline & $\begin{array}{l}\text { (Breast/mammary region) }+ \\
\text { regional lymph nodes }\end{array}$ & $43(42.6)$ \\
\hline \multirow{3}{*}{ RT technique } & 2D RT & $80(79.2)$ \\
\hline & 3D CRT & $14(13.9)$ \\
\hline & Electrons & $7(6.9)$ \\
\hline \multirow{2}{*}{ Hypertension } & Yes & $29(28.7)$ \\
\hline & No & $72(71.3)$ \\
\hline \multirow{2}{*}{ Hyperlipidemia } & Yes & $21(20.8)$ \\
\hline & No & $80(79.2)$ \\
\hline
\end{tabular}

TABLE 1: Continued.

\begin{tabular}{lcc}
\hline Characteristic & Category/Unit & $N(\%)$ \\
\hline \multirow{2}{*}{ Smoking } & Yes & $16(15.8)$ \\
& No & $85(84.2)$ \\
Diabetes & Yes & $1(1.0)$ \\
\multirow{2}{*}{ Body mass index } & No & $100(99.0)$ \\
& $\mathrm{kg} / \mathrm{m}^{2}$ & 27.1 \\
\end{tabular}

${ }^{*}$ median (25\%-75\%). 2D RT: two-dimensional radiotherapy; 3D CRT: threedimensional conformal radiotherapy; AC: doxorubicin, cyclophosphamide; BSA: body surface area calculated according to the Du Bois formula; EC: epirubicin, cyclophosphamide; FAC: 5-fluorouracil, doxorubicin, cyclophosphamide; FEC: 5-fluorouracil, epirubicin, and cyclophosphamide; Gy: Gray; RT: radiotherapy.

TABLe 2: Markers of cardiac side effects of breast cancer therapy.

\begin{tabular}{lcc}
\hline Marker & Category/Unit & $N(\%)$ \\
\hline Initial LVEF & $\%$ & $65(60-70)^{*}$ \\
Absolute change in LVEF & $\%$ & $3(-3 \text { do } 9)^{*}$ \\
LVEF reduction & No & $92(91.1)$ \\
NT-proBNP & Yes & $9(8.9)$ \\
NT-proBNP & ng/l & $90(56-157)^{*}$ \\
& $<125$ ng/l & $65(64.4)$ \\
NYHA & $\geq 125$ ng/l & $36(35.6)$ \\
& Class 1 & $84(83.2)$ \\
\hline
\end{tabular}

*median (25\%-75\%). LVEF: left ventricular ejection fraction; NT-proBNP: N-terminal pro B-type natriuretic peptide; NYHA: New York Heart Association. Absolute change in LVEF was calculated as the difference between LVEF after completed adjuvant radiotherapy and trastuzumab treatment, and LVEF before radiotherapy. LVEF reduction was classified as a decrease of $L V E F$ for $\geq 10 \%$ or a final value of $L V E F<50 \%$.

and RT have been associated with increased risk of cardiac adverse events [18]. In our study, more than one third of patients exhibited signs of cardiotoxicity after treatment. Differences in systemic therapy or RT parameters among patients were not associated with any of the investigated cardiotoxicity parameters. Among other characteristics, the most important clinical predictor of increased NT-proBNP was higher age, which is consistent with other studies and is reflected also in reference ranges for healthy individuals [50]. BMI has been reported to be associated with NTproBNP [50], but we did not observe any association with factors related to cardiovascular diseases such as BMI, smoking, hyperlipidemia or hypertension, or other clinical parameters. On the other hand, presence of hyperlipidemia and higher BMI were associated with mild symptoms of heart failure according to NYHA classification. Interestingly, none of the patients' or treatment characteristics were associated with important LVEF reduction.

Our results suggest that $P O N 1$ genetic variability was the most important predictor of treatment-related cardiotoxicity in HER2-positive early breast cancer patients. The key observation was the association of PON1 polymorphisms 
TABLE 3: Association of polymorphisms in antioxidative genes with NT-proBNP levels.

\begin{tabular}{|c|c|c|c|c|c|c|c|c|c|}
\hline SNP & Genotype & $\begin{array}{c}\text { NT-proBNP } \\
\text { median }(25-75 \%)\end{array}$ & $P$ & $\begin{array}{c}\text { NT-proBNP } \\
<125 \mathrm{ng} / \mathrm{l}, N(\%)\end{array}$ & $\begin{array}{c}\text { NT-proBNP } \\
\geq 125 \mathrm{ng} / \mathrm{l}, \mathrm{N}(\%)\end{array}$ & $\begin{array}{c}\text { OR } \\
(95 \% \mathrm{CI}) \\
\end{array}$ & $P$ & $\begin{array}{c}\text { OR } \\
(95 \% \mathrm{CI})_{\mathrm{adj}}\end{array}$ & $P_{\text {adj }}$ \\
\hline PON1 & AA & $126(59-200)$ & & $21(50.0)$ & $21(50.0)$ & Reference & & Reference & \\
\hline rs 854560 & $\mathrm{AT}+\mathrm{TT}$ & $79(51-125)$ & 0.048 & $44(74.6)$ & $15(25.4)$ & $0.34(0.15-0.79)$ & 0.012 & $0.35(0.15-0.83)$ & 0.017 \\
\hline PON1 & AA & $79(47.75-118.5)$ & & $43(79.6)$ & $11(20.4)$ & Reference & & Reference & \\
\hline rs 662 & $\mathrm{AG}+\mathrm{GG}$ & $135(60-193)$ & 0.007 & $22(46.8)$ & $25(53.2)$ & $4.44(1.85-10.66)$ & 0.001 & $5.41(2.12-13.78)$ & $<0.001$ \\
\hline GSTP1 & $\mathrm{CC}$ & $92(58-156)$ & & $53(63.9)$ & $30(36.1)$ & Reference & & Reference & \\
\hline rs1138272 & $\mathrm{CT}+\mathrm{TT}$ & $78.5(36-165.5)$ & 0.407 & $12(66.7)$ & $6(33.3)$ & $0.88(0.30-2.56)$ & 0.821 & $0.71(0.23-2.16)$ & 0.545 \\
\hline GSTP1 & GG & $122(65.75-174.5)$ & & $24(54.5)$ & $20(45.5)$ & Reference & & Reference & \\
\hline rs 1695 & $\mathrm{GA}+\mathrm{AA}$ & $77(48.5-144)$ & 0.101 & $41(71.9)$ & $16(28.1)$ & $0.47(0.21-1.07)$ & 0.073 & $0.36(0.15-0.88)$ & 0.026 \\
\hline CAT & GG & $88(52.5-148.25)$ & & $42(65.6)$ & $22(34.4)$ & Reference & & Reference & \\
\hline rs1001179 & $\mathrm{GA}+\mathrm{AA}$ & $96(56-182)$ & 0.680 & $23(62.2)$ & $14(37.8)$ & $1.16(0.50-2.70)$ & 0.762 & $0.99(0.41-2.36)$ & 0.973 \\
\hline HIF1A & $\mathrm{CC}$ & $91(56-154.5)$ & & $56(63.6)$ & $32(36.4)$ & Reference & & Reference & \\
\hline rs1154965 & $\mathrm{CT}+\mathrm{TT}$ & $79(54.5-180)$ & 0.666 & $9(69.2)$ & $4(30.8)$ & $0.78(0.22-2.73)$ & 0.695 & $0.87(0.24-3.13)$ & 0.827 \\
\hline
\end{tabular}

Adj: adjusted for age; CI: confidence interval; NT-proBNP: N-terminal pro B-type natriuretic peptide; OR: odds ratio.

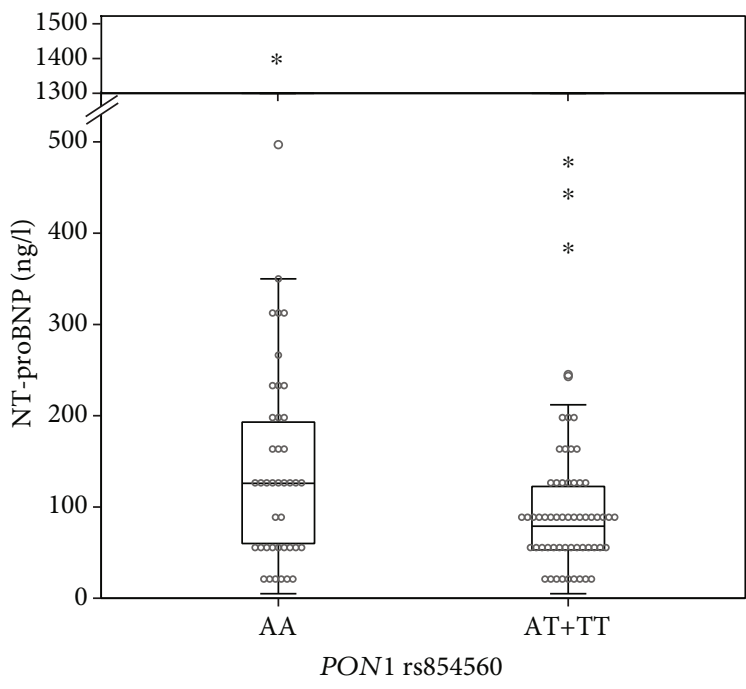

(a)

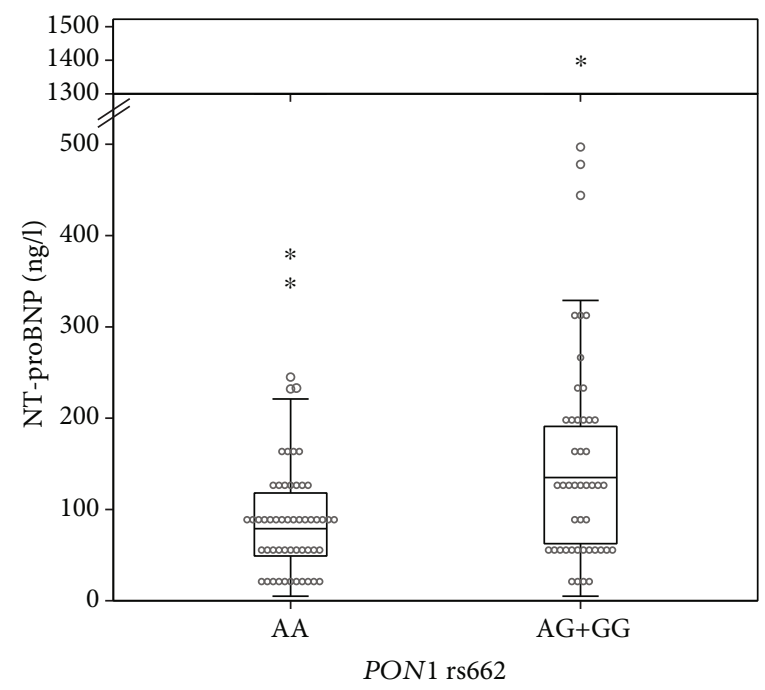

(b)

FIgURe 1: The association of PON1 rs854560 (a) and PON1 rs662 (b) polymorphisms with N-terminal pro B-type natriuretic peptide (NTproBNP) levels after adjuvant radiotherapy in HER2-positive breast cancer patients.

with NT-proBNP: polymorphic PON1 rs854560 T allele was associated with lower NT-proBNP levels, while PON1 polymorphic rs662 $\mathrm{G}$ allele was associated with higher NTproBNP levels. Additionally, an even larger association with increased NT-proBNP was observed in PON1 AG haplotype combining both normal rs854560 and polymorphic rs662 allele associated with higher NT-proBNP in single SNP analysis.

PON1 is a plasma enzyme located in HDL that has antioxidative, antiatherosclerotic, and anti-inflammatory role [51]. PON1 inhibits LDL oxidation, prevents accumulation of oxidized LDL, and stimulates cholesterol efflux from macrophages $[51,52]$. PON1 activity is inversely correlated with cardiovascular diseases $[45,51,52]$. Several functional polymorphisms were identified in the PON1 gene. PON1 rs854560 is a nonsynonymous SNP, and the leucine to methi- onine substitution was previously associated with increased enzyme activity and serum concentration [45, 53, 54]. PON1 rs662 is also a nonsynonymous SNP, leading to a glutamine to arginine substitution with the biggest impact on enzyme activity [53]. Polymorphic rs662 G allele results in lower enzymatic activity that limits PON1 capacity for lipid peroxide hydrolysis and therefore less effectively inhibits LDL oxidation $[45,54]$. Interestingly, rs662 has been associated with a substrate-specific change in enzyme activity: the polymorphic allele was associated with increased paraoxonase activity, while hydrolytic activity towards other substrates was lower $[45,53,55]$. PON1 rs662 was also associated with increased LDL and decreased HDL concentrations [56, 57]. The association of PON1 rs 854560 with lipoprotein levels is less pronounced and might also vary in different pathologies [51]. This data is in concordance with 


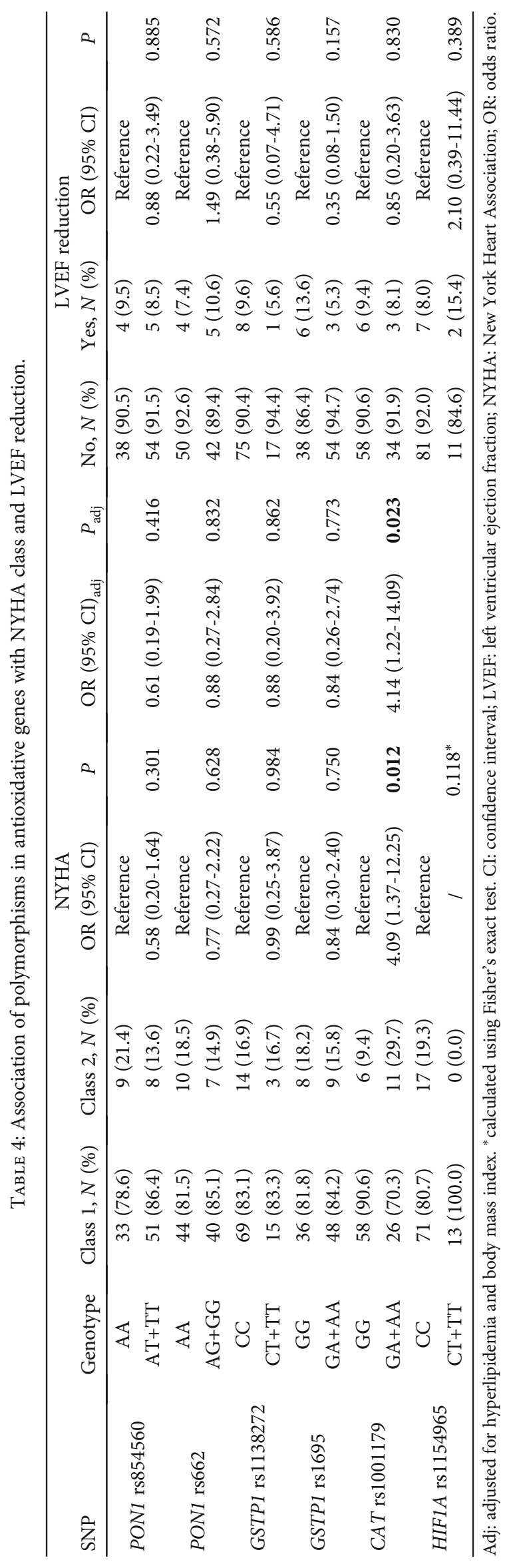


our results, as the polymorphic rs854560 T allele, associated with higher enzyme activity, was also associated with lower cardiotoxicity, while the polymorphic rs662 G allele, associated with lower enzyme activity, was associated with increased cardiotoxicity in our study.

A lot of studies also investigated the influence of PON1 polymorphisms on the risk of developing different cardiovascular diseases. Generally, PON1 rs662 was associated with slightly increased cardiovascular disease risk, especially in the recessive genetic model, while PON1 rs854560 was associated with somewhat decreased risk in meta-analyses [5862 ], consistent with our results. However, some studies found no significant associations and differences were observed among different populations [58, 59]. On the other hand, several studies also investigated the role of PON1 polymorphisms in cancer risk. Despite discrepancies among different studies, latest meta-analyses suggest rs662 is associated with lower breast cancer risk, while rs854560 is associated with increased breast cancer risk [63-66]. These results also suggest rs662 and rs854560 have an opposite effect, but further studies are needed as different results were observed in other cancer types [65].

Studies suggest serum PON1 level and its activity are lower in cancer patients, including breast cancer [67-70], but only a few studies investigated the role of PON1 in cancer treatment response or toxicity. So far, no studies investigated the association of PON1 with response to trastuzumab or anthracyclines, while a few studies focusing on RT were already published $[55,69,70]$. In breast cancer patients treated with adjuvant RT, PON1 concentration and activity increased after RT with significant differences observed among different molecular subtypes [70]. In luminal B (HER2-positive) subtype, PON1 concentration after RT was lower compared to luminal A subtype. HER2 expression was also associated with altered expression of other antioxidative enzymes, which could modify the risk for cardiotoxicity [70, 71]. In lung cancer, as well as head and neck cancer patients treated with RT, PON1 concentration also increased after RT [69]. Additionally, in patients with nasopharyngeal carcinoma, the combination of PON1 rs662 and another polymorphism, rs705379 (c.-108C>T) was associated with carotid atherosclerosis after RT of the neck, while PON1 rs854560 was not investigated [55]. In contrast to other studies, rs662 was associated with lower carotid plaque scores, which could be partly due to differences in activities observed for different substrates [55]. Better evaluation of $P O N 1$ genetic variability, concentration or activity is therefore needed to improve the understanding of PON1 role in cardiovascular disease and especially in treatment-related cardiotoxicity.

In our study, polymorphic GSTP1 rs1695 A allele was also significantly associated with lower risk for increased NT-proBNP after accounting for age. GSTP1 is involved in detoxification of xenobiotics through conjugation with glutathione [72]. GSTP1 rs1695 is a nonsynonymous SNP that leads to lower enzyme activity [73]. Several studies investigated the role of GSTP1 rs1695 in breast cancer susceptibility or response to treatment [73-79]. Latest meta-analyses suggest this SNP might contribute to increased breast cancer risk; however, the association was significant only in specific populations [73, 76, 77]. GSTP1 polymorphisms with lower enzyme activity were also proposed to be associated with better treatment outcome [78, 79], and based on meta-analysis results, GSTP1 rs1695 could serve as a predictor of response to anthracycline-based chemotherapy [78]. Additionally, this could also lead to altered risk of treatment-related toxicity. In a previous study, GSTP1 rs1695 was not associated with LVEF reduction after treatment with anthracyclines, consistent with our results, while NT-proBNP was not evaluated [80]. No studies investigated the association of GSTP1 with cardiotoxicity of treatment with RT or trastuzumab. However, rs1695 was previously associated with increased heart failure and coronary artery disease risk [81, 82]. Apart from its role in response to oxidative stress and lipid peroxidation, GSTP1 may also affect different signalling pathways, which could contribute to the observed association with heart disease [81]. Additional studies are needed to better understand the role of GSPT1 and its potential interaction with other clinical parameters in cardiotoxicity of breast cancer treatment, especially in patients treated with RT.

Carriers of at least one polymorphic CAT rs1001179 A allele were significantly more likely to exhibit mild symptoms of heart failure according to NYHA classification in our study, even after taking into account hyperlipidemia and BMI. This association was also observed in univariable analysis in the subgroup of left-sided breast cancer patients. In these cases, the heart lies directly below the target tissue for irradiation. CAT rs1001179 is located in the promoter region of the gene and was previously associated with lower expression and activity of catalase and thus could confer worse defence against oxidative stress $[43,83]$. Overall, the role of $C A T$ genetic variability in breast cancer is not well known, with previous studies suggesting rs1001179 is not associated with breast cancer risk [84]. Only a handful of studies have previously investigated the role of CAT genetic variability in cardiotoxicity of anthracyclines, while so far, no study has investigated cardiotoxicity of treatment with RT or trastuzumab [85-87]. In one study, intronic SNP CAT rs10836235 was associated with cardiac damage in childhood acute leukemia patients treated with anthracyclines, but no significant association with CAT rs1001179 was observed [85]. GWAS and meta-analyses did not identify catalase as a risk factor for cardiotoxicity of anthracyclines [86, 87]. Studies investigating the role of CAT polymorphisms in cardiovascular disease are also scarce; however, CAT rs1001179 was not a risk factor for coronary artery disease in a recent study [88]. Further studies are therefore needed to elucidate the role of catalase in the development of cardiotoxicity of breast cancer treatment after different treatment modalities.

The main limitation of our study was its small sample size. However, we had clear inclusion and exclusion criteria and thus included a clinically well-defined study group of patients with a HER2-positive breast cancer subtype with thorough evaluation of cardiotoxicity parameters. According to the available literature, we were the first to evaluate the role of genetic variability in cardiotoxicity after adjuvant RT in HER2-positive breast cancer patients. Another limitation of our study is the fact that we had to exclude SOD2 rs4880 from 
the analysis as it was not in agreement with HWE. As SOD2 rs4880 was marginally associated with breast cancer risk in Caucasians, this could contribute to the observed deviation from HWE [89]. Still, we were among the first to assess the influence of genetic variability of several antioxidative genes on cardiotoxicity of breast cancer treatment and the first to show that especially PON1 polymorphisms could contribute to the occurrence of cardiac adverse events. As our patients were treated with different treatment modalities that all contribute to cardiotoxicity, studies on patients treated only with RT or with only one type of systemic therapy could help elucidate the role of the investigated polymorphisms. Larger studies are therefore needed to further validate our results.

\section{Conclusions}

In conclusion, our study indicates that functional polymorphisms in antioxidative genes might serve as biomarkers of treatment-related cardiotoxicity in breast cancer patients. Better understanding of adverse events could improve patient management and affect the health and quality of life of breast cancer patients. In the future, genetic markers could contribute to the personalization of RT and systemic therapy in breast cancer patients.

\section{Data Availability}

The data used to support the findings of this study are included within the article and supplementary information file.

\section{Conflicts of Interest}

The authors declare no conflict of interest.

\section{Acknowledgments}

This work was financially supported by the Slovenian Research Agency [ARRS Grant Nos. J3-1753, P1-0170, and P3-0321].

\section{Supplementary Materials}

Supplementary Table 1: genotype frequencies of investigated polymorphisms. (Supplementary Materials)

\section{References}

[1] EBCTCG (Early Breast Cancer Trialists' Collaborative Group), P. McGale, C. Taylor et al., "Effect of radiotherapy after mastectomy and axillary surgery on 10-year recurrence and 20year breast cancer mortality: meta-analysis of individual patient data for 8135 women in 22 randomised trials," Lancet, vol. 383, no. 9935, pp. 2127-2135, 2014.

[2] M. Clarke, R. Collins, S. Darby et al., "Effects of radiotherapy and of differences in the extent of surgery for early breast cancer on local recurrence and 15-year survival: an overview of the randomised trials," Lancet, vol. 366, no. 9503, pp. 2087-2106, 2005.

[3] I. Meattini, M. Guenzi, A. Fozza et al., "Overview on cardiac, pulmonary and cutaneous toxicity in patients treated with adjuvant radiotherapy for breast cancer," Breast Cancer, vol. 24, no. 1, pp. 52-62, 2017.

[4] Q. Zhu, Y. M. Kirova, L. Cao, A. Arsene-Henry, and J. Chen, "Cardiotoxicity associated with radiotherapy in breast cancer: a question-based review with current literatures," Cancer Treatment Reviews, vol. 68, pp. 9-15, 2018.

[5] S. C. Darby, M. Ewertz, P. McGale et al., "Risk of ischemic heart disease in women after radiotherapy for breast cancer," New England Journal of Medicine, vol. 368, no. 11, pp. 987998, 2013.

[6] T. Marinko, J. Dolenc, and C. Bilban-Jakopin, "Cardiotoxicity of concomitant radiotherapy and trastuzumab for early breast cancer," Radiology and Oncology, vol. 48, no. 2, pp. 105-112, 2014.

[7] F. Cardoso, S. Kyriakides, S. Ohno et al., "Early breast cancer: ESMO Clinical Practice Guidelines for diagnosis, treatment and follow-up ${ }^{\dagger}, "$ Annals of Oncology, vol. 30, no. 8, pp. 1194-1220, 2019.

[8] T. Marinko, S. Borstnar, R. Blagus, J. Dolenc, and C. BilbanJakopin, "Early cardiotoxicity after adjuvant concomitant treatment with radiotherapy and trastuzumab in patients with breast cancer," Radiology and Oncology, vol. 52, no. 2, pp. 204212,2018

[9] H. J. Burstein, G. Curigliano, S. Loibl et al., "Estimating the benefits of therapy for early-stage breast cancer: the St. Gallen International Consensus Guidelines for the primary therapy of early breast cancer 2019," Annals of Oncology, vol. 30, no. 10, pp. 1541-1557, 2019.

[10] Early Breast Cancer Trialists' Collaborative Group (EBCTCG), R. Peto, C. Davies et al., "Comparisons between different polychemotherapy regimens for early breast cancer: meta-analyses of long-term outcome among 100000 women in 123 randomised trials," Lancet, vol. 379, no. 9814, pp. 432-444, 2012.

[11] P. P. Advani, K. V. Ballman, T. J. Dockter, G. Colon-Otero, and E. A. Perez, "Long-term cardiac safety analysis of NCCTG N9831 (Alliance) adjuvant trastuzumab trial," Journal of Clinical Oncology, vol. 34, no. 6, pp. 581-587, 2016.

[12] C. A. Geisberg and D. B. Sawyer, "Mechanisms of anthracycline cardiotoxicity and strategies to decrease cardiac damage," Current hypertension reports, vol. 12, no. 6, pp. 404-410, 2010.

[13] M. L. Henry, J. Niu, N. Zhang, S. H. Giordano, and M. ChavezMacGregor, "Cardiotoxicity and cardiac monitoring among chemotherapy-treated breast cancer patients," JACC Cardiovascular imaging, vol. 11, no. 8, pp. 1084-1093, 2018.

[14] M. Fiúza, "Cardiotoxicity associated with trastuzumab treatment of HER2+ breast cancer," Advances in Therapy, vol. 26, 1, pp. S9-S17, 2009.

[15] M. S. Ewer and S. M. Lippman, "Type II chemotherapy-related cardiac dysfunction: time to recognize a new entity," Journal of Clinical Oncology, vol. 23, no. 13, pp. 2900-2902, 2005.

[16] A. H. Blaes, P. Thavendiranathan, and J. Moslehi, "Cardiac toxicities in the era of precision medicine: underlying risk factors, targeted therapies, and cardiac biomarkers," American Society of Clinical Oncology educational book, vol. 38, pp. 764-774, 2018.

[17] G. Curigliano, D. Lenihan, M. Fradley et al., "Management of cardiac disease in cancer patients throughout oncological treatment: ESMO consensus recommendations," Annals of Oncology, vol. 31, no. 2, pp. 171-190, 2020.

[18] L. Michel, T. Rassaf, and M. Totzeck, "Biomarkers for the detection of apparent and subclinical cancer therapy-related 
cardiotoxicity," Journal of Thoracic Disease, vol. 10, Suppl 35, pp. S4282-s4295, 2018.

[19] S. Goel, R. J. Simes, and J. M. Beith, "Exploratory analysis of cardiac biomarkers in women with normal cardiac function receiving trastuzumab for breast cancer," Asia-Pacific journal of clinical oncology, vol. 7, no. 3, pp. 276-280, 2011.

[20] N. Ponde, I. Bradbury, M. Lambertini et al., "Cardiac biomarkers for early detection and prediction of trastuzumab and/or lapatinib-induced cardiotoxicity in patients with HER2-positive early-stage breast cancer: a NeoALTTO substudy (BIG 1-06)," Breast Cancer Research and Treatment, vol. 168, no. 3, pp. 631-638, 2018.

[21] S. Geiger, V. Lange, P. Suhl, V. Heinemann, and H. J. Stemmler, "Anticancer therapy induced cardiotoxicity: review of the literature," Anti-Cancer Drugs, vol. 21, no. 6, pp. 578-590, 2010.

[22] D. Zardavas, T. M. Suter, D. van Veldhuisen et al., "Role of troponins I and $\mathrm{T}$ and N-terminal prohormone of brain natriuretic peptide in monitoring cardiac safety of patients with early-stage human epidermal growth factor receptor 2positive breast cancer receiving trastuzumab: a herceptin adjuvant study cardiac marker substudy," Journal of Clinical Oncology, vol. 35, no. 8, pp. 878-884, 2017.

[23] A. H. M. de Vries Schultink, A. H. Boekhout, J. A. Gietema et al., "Pharmacodynamic modeling of cardiac biomarkers in breast cancer patients treated with anthracycline and trastuzumab regimens," Journal of pharmacokinetics and pharmacodynamics, vol. 45, no. 3, pp. 431-442, 2018.

[24] E. Birben, U. M. Sahiner, C. Sackesen, S. Erzurum, and O. Kalayci, "Oxidative stress and antioxidant defense," The World Allergy Organization journal, vol. 5, no. 1, pp. 9-19, 2012.

[25] G. Pizzino, N. Irrera, M. Cucinotta et al., "Oxidative stress: harms and benefits for human health," Oxidative medicine and cellular longevity, vol. 2017, 13 pages, 2017.

[26] W. Kim, S. Lee, D. Seo et al., "Cellular stress responses in radiotherapy," Cells, vol. 8, no. 9, p. 1105, 2019.

[27] Z. Ping, Y. Peng, H. Lang et al., "Oxidative stress in radiationinduced cardiotoxicity," Oxidative medicine and cellular longevity, vol. 2020, 15 pages, 2020.

[28] N. Prathumsap, K. Shinlapawittayatorn, S. C. Chattipakorn, and N. Chattipakorn, "Effects of doxorubicin on the heart: from molecular mechanisms to intervention strategies," European Journal of Pharmacology, vol. 866, p. 172818, 2020.

[29] N. Mohan, J. Jiang, and W. J. Wu, "Implications of autophagy and oxidative stress in trastuzumab-mediated cardiac toxicities," Austin pharmacology \& pharmaceutics, vol. 2, no. 1, 2017.

[30] L. Rochette, C. Guenancia, A. Gudjoncik et al., "Anthracyclines/trastuzumab: new aspects of cardiotoxicity and molecular mechanisms," Trends in Pharmacological Sciences, vol. 36, no. 6, pp. 326-348, 2015.

[31] I. Dogan, A. Cumaoglu, A. Aricioglu, and A. Ekmekci, "Inhibition of ErbB2 by herceptin reduces viability and survival, induces apoptosis and oxidative stress in Calu-3 cell line," Molecular and Cellular Biochemistry, vol. 347, no. 1-2, pp. 41-51, 2011.

[32] D. W. Nebert and V. Vasiliou, "Analysis of the glutathione Stransferase (GST) gene family," Human Genomics, vol. 1, no. 6, pp. 460-464, 2004.

[33] T. Fukai and M. Ushio-Fukai, "Superoxide dismutases: role in redox signaling, vascular function, and diseases," Antioxidants \& redox signaling, vol. 15, no. 6, pp. 1583-1606, 2011.
[34] A. Nandi, L. J. Yan, C. K. Jana, and N. Das, "Role of catalase in oxidative stress- and age-associated degenerative diseases," Oxidative medicine and cellular longevity, vol. 2019, 2019.

[35] D. Litvinov, H. Mahini, and M. Garelnabi, "Antioxidant and anti-inflammatory role of paraoxonase 1: implication in arteriosclerosis diseases," North American journal of medical science, vol. 4, no. 11, pp. 523-532, 2012.

[36] Z. N. Rabbani, J. Mi, Y. Zhang et al., "Hypoxia inducible factor 1alpha signaling in fractionated radiation-induced lung injury: role of oxidative stress and tissue hypoxia," Radiation Research, vol. 173, no. 2, pp. 165-174, 2010.

[37] B. J. Moeller, Y. Cao, C. Y. Li, and M. W. Dewhirst, "Radiation activates HIF-1 to regulate vascular radiosensitivity in tumors: role of reoxygenation, free radicals, and stress granules," Cancer Cell, vol. 5, no. 5, pp. 429-441, 2004.

[38] L. Slemc and T. Kunej, "Transcription factor HIF1A: downstream targets, associated pathways, polymorphic hypoxia response element (HRE) sites, and initiative for standardization of reporting in scientific literature," Tumour Biology, vol. 37, no. 11, pp. 14851-14861, 2016.

[39] I. Gladek, J. Ferdin, S. Horvat, G. A. Calin, and T. Kunej, "HIF1A gene polymorphisms and human diseases: graphical review of 97 association studies," Genes, Chromosomes and Cancer, vol. 56, no. 6, pp. 439-452, 2017.

[40] G. C. Barnett, C. E. Coles, R. M. Elliott et al., "Independent validation of genes and polymorphisms reported to be associated with radiation toxicity: a prospective analysis study," The Lancet Oncology, vol. 13, no. 1, pp. 65-77, 2012.

[41] T. Rattay and C. J. Talbot, "Finding the genetic determinants of adverse reactions to radiotherapy," Clinical Oncology, vol. 26, no. 5, pp. 301-308, 2014.

[42] P. Seibold, S. Behrens, P. Schmezer et al., "XRCC1 polymorphism associated with late toxicity after radiation therapy in breast cancer patients," International Journal of Radiation Oncology • Biology • Physics, vol. 92, no. 5, pp. 1084-1092, 2015.

[43] K. Liu, X. Liu, M. Wang et al., "Two common functional catalase gene polymorphisms (rs1001179 and rs794316) and cancer susceptibility: evidence from 14,942 cancer cases and 43,285 controls," Oncotarget, vol. 7, no. 39, pp. 62954-62965, 2016.

[44] S. W. Kang, "Superoxide dismutase 2 gene and cancer risk: evidence from an updated meta-analysis," International journal of clinical and experimental medicine, vol. 8, no. 9, pp. 14647-14655, 2015.

[45] N. Shunmoogam, P. Naidoo, and R. Chilton, "Paraoxonase (PON)-1: a brief overview on genetics, structure, polymorphisms and clinical relevance," Vascular health and risk management, vol. 14, pp. 137-143, 2018.

[46] E. Matos, B. Zakotnik, and C. G. Kuhar, "Effectiveness of adjuvant trastuzumab in daily clinical practice," Radiology and Oncology, vol. 48, no. 4, pp. 403-407, 2014.

[47] C. Bredy, M. Ministeri, A. Kempny et al., "New York Heart Association (NYHA) classification in adults with congenital heart disease: relation to objective measures of exercise and outcome," European heart journal. Quality of care \& clinical outcomes, vol. 4, no. 1, pp. 51-58, 2018.

[48] Z. Cao, Y. Jia, and B. Zhu, "BNP and NT-proBNP as diagnostic biomarkers for cardiac dysfunction in both clinical and forensic medicine," International journal of molecular sciences, vol. 20, no. 8, p. 1820, 2019. 
[49] D. A. Tregouet and V. Garelle, "A new JAVA interface implementation of THESIAS: testing haplotype effects in association studies," Bioinformatics, vol. 23, no. 8, pp. 1038-1039, 2007.

[50] M. G. Fradley, M. G. Larson, S. Cheng et al., "Reference limits for N-terminal-pro-B-type natriuretic peptide in healthy individuals (from the Framingham Heart Study)," American Journal of Cardiology, vol. 108, no. 9, pp. 1341-1345, 2011.

[51] K. Kowalska, E. Socha, and H. Milnerowicz, "Review: the role of paraoxonase in cardiovascular diseases," Annals of Clinical Laboratory Science, vol. 45, no. 2, pp. 226-233, 2015.

[52] M. Rosenblat, L. Gaidukov, O. Khersonsky et al., "The catalytic histidine dyad of high density lipoprotein-associated serum paraoxonase-1 (PON1) is essential for PON1-mediated inhibition of low density lipoprotein oxidation and stimulation of macrophage cholesterol efflux," Journal of Biological Chemistry, vol. 281, no. 11, pp. 7657-7665, 2006.

[53] K. Huen, L. Barcellos, K. Beckman, S. Rose, B. Eskenazi, and N. Holland, "Effects of PON polymorphisms and haplotypes on molecular phenotype in Mexican-American mothers and children," Environmental and Molecular Mutagenesis, vol. 52, no. 2, pp. 105-116, 2011.

[54] B. Mackness, M. I. Mackness, S. Arrol, W. Turkie, and P. N. Durrington, "Effect of the human serum paraoxonase 55 and 192 genetic polymorphisms on the protection by high density lipoprotein against low density lipoprotein oxidative modification," FEBS Letters, vol. 423, no. 1, pp. 57-60, 1998.

[55] C. Yuan, S. P. Yip, V. W. Wu, D. L. Kwong, I. W. Cheuk, and M. Ying, "Association between genetic polymorphisms and carotid atherosclerosis in patients treated with radiotherapy for nasopharyngeal carcinoma," Radiation oncology, vol. 10, p. 39, 2015.

[56] A. Vaisi-Raygani, H. Ghaneialvar, Z. Rahimi et al., "Paraoxonase Arg 192 allele is an independent risk factor for threevessel stenosis of coronary artery disease," Molecular Biology Reports, vol. 38, no. 8, pp. 5421-5428, 2011.

[57] N. Pérez-Herrera, C. May-Pech, I. Hernández-Ochoa et al., "PON1Q192R polymorphism is associated with lipid profile in Mexican men with Mayan ascendancy," Experimental and Molecular Pathology, vol. 85, no. 2, pp. 129-134, 2008.

[58] Q. H. Zeng and J. Zeng, "A meta-analysis on relationship between paraoxonase 1 polymorphisms and atherosclerotic cardiovascular diseases," Life Sciences, vol. 232, 2019.

[59] Z. Zhang, J. K. Ou, P. L. Cai, B. Niu, and J. Li, “Association between the PON1 Q192R polymorphism and coronary heart disease in Chinese: a meta-analysis," Medicine, vol. 97, no. 26, 2018.

[60] D. Lawlor, S. Ebrahim, I. M. Day et al., "The association of the PON1 Q192R polymorphism with coronary heart disease: findings from the British Womens Heart and Health prospective cohort study and a meta-analysis," European Heart Journal, vol. 25, p. 531, 2004.

[61] M. S. Wang, X. L. Lang, L. J. Zou, S. D. Huang, and Z. Y. Xu, "Four genetic polymorphisms of paraoxonase gene and risk of coronary heart disease: a meta-analysis based on 88 case-control studies," Atherosclerosis, vol. 214, no. 2, pp. 377-385, 2011.

[62] J. G. Wheeler, B. D. Keavney, H. Watkins, R. Collins, and J. Danesh, "Four paraoxonase gene polymorphisms in 11,212 cases of coronary heart disease and 12,786 controls: metaanalysis of 43 studies," Lancet, vol. 363, no. 9410, pp. 689695, 2004.
[63] L. Chen, W. Lu, L. Fang et al., "Association between L55M polymorphism in Paraoxonase 1 and cancer risk: a metaanalysis based on 21 studies," OncoTargets and therapy, vol. 9, pp. 1151-1158, 2016.

[64] X. Pan, L. Huang, M. Li et al., "The association between PON1 (Q192R and L55M) gene polymorphisms and risk of cancer: a meta-analysis based on 43 studies," BioMed research international, vol. 2019, 14 pages, 2019.

[65] M. Zhang, H. Xiong, L. Fang et al., "Paraoxonase 1 (PON1) Q192R gene polymorphism and cancer risk: a meta-analysis based on 30 publications," Asian Pacific journal of cancer prevention: APJCP, vol. 16, no. 10, pp. 4457-4463, 2015.

[66] M. Saadat, "Paraoxonase 1 genetic polymorphisms and susceptibility to breast cancer: a meta-analysis," Cancer Epidemiology, vol. 36, no. 2, pp. e101-e103, 2012.

[67] Z. Q. Samra, S. Pervaiz, S. Shaheen, N. Dar, and M. A. Athar, "Determination of oxygen derived free radicals producer (xanthine oxidase) and scavenger (paraoxonase1) enzymes and lipid parameters in different cancer patients," Clinica y Laboratorio, vol. 57, no. 9-10, pp. 741-747, 2011.

[68] H. Kocoglu, Y. Okuturlar, M. Gunaldi et al., "Serum paraoxonase and arylesterase can be useful markers to predict neoadjuvant chemotherapy requirement in patients with breast cancer," Journal of Cancer Research and Therapeutics, vol. 14, no. 9, p. 362, 2018.

[69] E. Rodríguez-Tomàs, M. Murcia, M. Arenas et al., "Serum paraoxonase-1-related variables and lipoprotein profile in patients with lung or head and neck cancer: effect of radiotherapy," Antioxidants, vol. 8, no. 7, 2019.

[70] M. Arenas, A. García-Heredia, N. Cabré et al., "Effect of radiotherapy on activity and concentration of serum paraoxonase- 1 in breast cancer patients," PLoS One, vol. 12, no. 11, article e0188633, 2017.

[71] F. Belmonte, S. Das, P. Sysa-Shah et al., "ErbB2 overexpression upregulates antioxidant enzymes, reduces basal levels of reactive oxygen species, and protects against doxorubicin cardiotoxicity," American journal of physiology. Heart and circulatory physiology, vol. 309, no. 8, pp. H1271-H1280, 2015.

[72] J. Ma, S. L. Zhu, Y. Liu, X. Y. Huang, and D. K. Su, "GSTP1 polymorphism predicts treatment outcome and toxicities for breast cancer," Oncotarget, vol. 8, no. 42, pp. 72939-72949, 2017.

[73] L. F. Miao, X. H. Ye, and X. F. He, "Individual and combined effects of GSTM1, GSTT1, and GSTP1 polymorphisms on breast cancer risk: a meta-analysis and re-analysis of systematic meta-analyses," PLoS One, vol. 15, no. 3, article e0216147, 2020.

[74] L. N. Al-Eitan, D. M. Rababa'h, M. A. Alghamdi, and R. H. Khasawneh, "Association Of GSTM1, GSTT1 and GSTP1 polymorphisms with breast cancer among Jordanian women," OncoTargets and therapy, vol. 12, pp. 7757-7765, 2019.

[75] A. Farmohammadi, V. Arab-Yarmohammadi, and R. Ramzanpour, "Association analysis of rs1695 and rs1138272 variations in GSTP1 gene and breast cancer susceptibility," Asian Pacific journal of cancer prevention, vol. 21, no. 4, pp. 1167-1172, 2020.

[76] M. Kuang, W. Xu, C. X. Cao et al., "Glutathione S-transferase P1 rs1695 A>G polymorphism and breast cancer risk: evidence from a meta-analysis," Genetics and molecular research, vol. 15 , no. 2, 2016.

[77] Z. Song, C. Shao, C. Feng, Y. Lu, Y. Gao, and C. Dong, "Association of glutathione S-transferase T1, M1, and P1 
polymorphisms in the breast cancer risk: a meta-analysis," Therapeutics and Clinical Risk Management, vol. 12, pp. 763-769, 2016.

[78] X. Kong, Z. Li, and X. Li, “GSTP1, GSTM1, and GSTT1 polymorphisms as predictors of response to chemotherapy in patients with breast cancer: a meta-analysis," Cancer Chemotherapy and Pharmacology, vol. 78, no. 6, pp. 1163-1173, 2016.

[79] J. Liu, J. Luo, Y. Wang, L. Li, and S. Yang, "Predictive potential role of glutathione S-transferases polymorphisms on prognosis of breast cancer," International journal of clinical and experimental pathology, vol. 7, no. 12, pp. 8935-8940, 2014.

[80] D. Vivenza, M. Feola, O. Garrone, M. Monteverde, M. Merlano, and C. Lo Nigro, "Role of the renin-angiotensin-aldosterone system and the glutathione S-transferase $\mathrm{Mu}, \mathrm{Pi}$ and Theta gene polymorphisms in cardiotoxicity after anthracycline chemotherapy for breast carcinoma," International Journal of Biological Markers, vol. 28, no. 4, pp. e336-e347, 2013.

[81] D. Simeunovic, N. Odanovic, M. Pljesa-Ercegovac et al., "Glutathione transferase P1 polymorphism might be a risk determinant in heart failure," Disease Markers, vol. 2019, 11 pages, 2019.

[82] J. S. Bhatti, R. Vijayvergiya, B. Singh, and G. K. Bhatti, "Genetic susceptibility of glutathione S-transferase genes (GSTM1/T1 and $\mathrm{P} 1$ ) to coronary artery disease in Asian Indians," Annals of Human Genetics, vol. 82, no. 6, pp. 448-456, 2018.

[83] J. Ahn, "Associations between catalase phenotype and genotype: modification by epidemiologic factors," Cancer Epidemiology Biomarkers \& Prevention, vol. 15, no. 6, pp. 1217-1222, 2006.

[84] M. Saadat and S. Saadat, "Genetic polymorphism of CAT C$262 \mathrm{~T}$ and susceptibility to breast cancer, a case-control study and meta-analysis of the literatures," Pathology Oncology Research, vol. 21, no. 2, pp. 433-437, 2015.

[85] V. Rajić, R. Aplenc, M. Debeljak et al., "Influence of the polymorphism in candidate genes on late cardiac damage in patients treated due to acute leukemia in childhood," Leukemia and Lymphoma, vol. 50, no. 10, pp. 1693-1698, 2009.

[86] B. P. Schneider, F. Shen, L. Gardner et al., "Genome-wide association study for anthracycline-induced congestive heart failure," Clinical Cancer Research, vol. 23, no. 1, pp. 43-51, 2017.

[87] S. L. Leong, N. Chaiyakunapruk, and S. W. H. Lee, "Candidate gene association studies of anthracycline-induced cardiotoxicity: a systematic review and meta-analysis," Scientific Reports, vol. 7, 2017.

[88] H. L. Yeh, L. T. Kuo, F. C. Sung, and C. C. Yeh, “Association between polymorphisms of antioxidant gene (MnSOD, CAT, and GPx1) and risk of coronary artery disease," BioMed research international, vol. 2018, 8 pages, 2018.

[89] C. Wang, Y. Liu, J. Zhou et al., "There is no relationship between SOD2 Val-16Ala polymorphism and breast cancer risk or survival," Molecular and clinical oncology, vol. 7, no. 4, pp. 579-590, 2017. 